International Journal of Advanced Chemistry, $2(1)(2014) 12-16$
International Journal of Advanced Chemistry
Journal home page: $\begin{gathered}\text { www.sciencepubco.com/index.php/IJAC } \\ \text { doi: } 10.14419 / \text { ijac.v2il.1624 } \\ \text { Research Paper }\end{gathered}$

\title{
Molecular interaction studies in binary liquid mixture through ultrasonic measurements at 303.15k
}

\author{
N. Santhi*, J. Madhumitha \\ Department of Chemistry, Government Arts College, Chidambaram-608201, Tamil Nadu, India \\ *Corresponding author E-mail: nsaanthi@gmail.com
}

\begin{abstract}
The ultrasonic velocity $(U)$, density $(\rho)$ and viscosity $(\eta)$ have been measured for the binary liquid mixtures containing 2 -(p-methoxy phenylsulphonyl)-4-methyl sydnone in Ethanol at 303.15K. From these data some of acoustical parameters such as adiabatic compressibility $(\beta)$, free length (Lf), acoustic impedance $(Z)$, relative association (RA), relaxation strength (r), Rao's constant (R), molar compressibility $(\mathrm{W})$, relaxation time $(\tau)$, Vander waal's constant $(\mathrm{b})$, free volume $(\mathrm{Vf})$ and internal pressure $(\pi \mathrm{i})$ have been computed using the standard relations. The results have been discussed in terms of molecular interactions.
\end{abstract}

Keywords: Binary mixture, Molecular interaction, ultrasonic velocity, density, viscosity.

\section{Introduction}

In recent years, ultrasonic technique has become a powerful tool for studying the molecular behaviour of liquid mixtures1-3. This is because of its ability of characterizing physico-chemical behaviour of liquid medium4-6. The study of properties of liquid mixtures and solutions find direct applications in chemical and biochemical industries 7-8. Ultrasonic velocity and related thermodynamic parameters helps us for characterizing thermodynamic and physico-chemical aspects of binary liquid mixtures such as molecular association and dissociation9-10. Ultrasonic velocity together with density and viscosity data furnish wealth of information about the interaction between ions, dipoles, hydrogen bonding, multipolar and dispersive forces11-12. The ultrasonic velocity in a liquid is fundamentally related to the binding forces between the atoms or molecules and has been successfully employed in understanding the nature of molecular interactions in pure liquids, binary and ternary mixtures13-15.

The variation of ultrasonic velocity and related parameters throw much light upon the structural changes associated with the liquid mixtures having strongly interacting components 16 as well as weakly interacting components17. Molecular interaction studies can be carried out by both spectroscopic18-19 and nonspectroscopic20-21 techniques. The physical and chemical properties of liquid mixtures have been studied by number of workers22 23 and they correlated the non-linear variation of ultrasonic velocity, compressibility and other related parameters with structural changes occurring in a liquid as its concentration is varied in a liquid mixture.

Experimental:

Density:

The density of pure liquids and mixtures are measured using a $10 \mathrm{ml}$ specific gravity bottle. The measured density was calculated using the formula

$\rho 2=(w 2 / w 1) \rho 1$

Where, w1 is the weight of the distilled water
$\mathrm{W} 2$ is the weight of the experimental liquid

$\rho 1$ is the density of water

Viscosity:

The viscosity of the pure liquids and liquid mixtures are measured using an Ostwald's Viscometer calibrated with doubly distilled water. The Ostwald's Viscometer with the experimental liquid is immersed in a temperature controlled water bath at $303.15 \mathrm{~K}$. The digital stopwatch, with an accuracy of $+0.01 \mathrm{sec}$ was used to determine flow time of solutions. Using the flow times (t) and known of standard water sample, the viscosity of solvent and solutions were determined according the following equation:

$\eta 2=\eta 1(t 2 / t))(\rho 2 / \rho 1)$

Where

$\eta 1$ is the viscosity of water

t1 is the time of flow of water

$\rho 1$ is the density of water

$\eta 2$ is the viscosity of the binary mixture

$\mathrm{t} 2$ is the time of flow of the binary mixture

$\rho 2$ is the density of the binary mixture

Ultrasonic velocity:

The sound velocity of the liquid mixture have been measured using an ultrasonic interferometer (Mittal Enterprises, New Delhi) working at a fixed frequency of $2 \mathrm{MHZ}$.

The binary liquid mixture is filled in the measuring cell with quartz crystal and then micrometer was fixed. The circulation of water from the thermostat at $303.15 \mathrm{~K}$ was started and test solution in the cell is allowed to thermally equilibrate. The micrometer was rotated very slowly so as to obtain a maximum or minimum of anode current (n). A number of maximum reading of anode current were counted. The total distance (d) travel by the micrometer for $\mathrm{n}=10$, was read. The wave length $(\lambda)$ was determined according to the following equation:

$\lambda=2 \mathrm{~d} / \mathrm{n}$

The sound velocity (U) of solvent and solutions were calculated from the wavelength and frequency $(\mathrm{F})$ according to the following Equation:

$\mathrm{U}=\lambda \mathrm{F}$ 


\section{Theory:}

The ultrasonic velocity $(U)$, density $(\rho)$ and viscosity $(\eta)$ in pure liquids and liquid mixtures of various concentrations have been measured at $303.15 \mathrm{~K}$. Thermodynamic and acoustical parameters such as adiabatic compressibility $(\beta)$, free length (Lf), acoustic impedance (Z), relative association (RA), relaxation strength (r), Rao's constant $(\mathrm{R})$, molar compressibility $(\mathrm{W})$, relaxation time $(\tau)$, Vander waal's constant (b), free volume (Vf) and internal pressure $(\pi \mathrm{i})$ were determined using the observed values of velocity, density $\&$ viscosity using the standard relations given below.

1) The adiabatic compressibility $(\beta)$ has been calculated from sound velocity $(\mathrm{U})$ and the density $(\rho)$ of the medium using the relation

$\beta=1 / \mathrm{U} 2 \rho$

2) Intermolecular free length (Lf ) is calculated using the standard expression

$\mathrm{Lf}=\mathrm{K} \beta 1 / 2$

Where $\mathrm{K}$ is a Jacobson's constant $(=2.0965 \mathrm{X} 10-6)$

3) Acoustic impedance $(Z)$ was calculated by the equation $\mathrm{Z}=\mathrm{U} \rho$

Where $\rho$ is the density of the mixture and $U$ is the ultrasonic velocity of the mixture.

4) Molar compressibility or Wada's constant (W) can be calculated by the following equation

$\mathrm{W}=(\mathrm{M} / \rho) \beta-1 / 7$

Where $\mathrm{M}$ is the molecular weight of the solution which can be calculated according to the equation

$\mathrm{M}=\mathrm{M} 1 \mathrm{X} 1+\mathrm{M} 2 \mathrm{X} 2$

Where $\mathrm{X} 1$ and $\mathrm{X} 2$ are mole fractions of solvent and solute, respectively. M1 and M2 are the molecular weights of the solvent and solute respectively.

5) The molar sound velocity or Rao's constant (R) was calculated by the equation

$\mathrm{R}=(\mathrm{M} / \rho) \mathrm{U} 1 / 3$

(6)

Where $\rho$ is the density and $\mathrm{U}$ is the ultrasonic velocity of the mixture.

6) The Relative association (RA) was calculated by the following equation

$\mathrm{RA}=\rho / \rho \mathrm{o}(\mathrm{Uo} / \mathrm{U}) 1 / 3$

Where U, Uo and $\rho$, $\rho$ o are ultrasonic velocities and densities of solution and solvent respectively.

7) Relaxation strength (r) was calculated by the following equation

$\mathrm{r}=1-[\mathrm{U} / \mathrm{U} \infty] 2$

Where $\mathrm{U}_{\infty}=1600 \mathrm{~m} / \mathrm{sec}$.

8) Vander Waals constant (b) was calculated by the following equation

$\mathrm{b}=(\mathrm{M} / \rho)[1-(\mathrm{RT} / \mathrm{MU} 2)\{(1+\mathrm{MU} 2 / 3 \mathrm{RT}) 1 / 2-1\}]$

Where $\mathrm{R}$ is the gas constant $(=8.314 \mathrm{JK}-1 \mathrm{~mol}-1)$ and $\mathrm{T}$ is the absolute temperature.

9) Viscous relaxation time $(\tau)$ was calculated by the following equation

$\tau=4 \eta / 3 \rho \mathrm{U} 2$

Where $\eta$ is the viscosity, $\rho$ is the density and $U$ is the ultrasonic velocity of the mixture.

10) Free volume (Vf) was calculated by the following equation

$\mathrm{Vf}=[\mathrm{MU} / \mathrm{K} \eta] 3 / 2$

Where $\mathrm{K}$ is a constant $(=4.28 \times 109)$

11) The Internal pressure $(\pi \mathrm{i})$ was calculated by the following equation

$\pi \mathrm{i}=\mathrm{bRT}[\mathrm{K \eta} / \mathrm{U}] 1 / 2 \rho 2 / 3 / \mathrm{M} 7 / 6$
Where $\mathrm{b}$ is the packing factor $(=2), \mathrm{K}$ is a constant (=4.28 X 109). The following Tables represent the measured parameters viz ultrasonic velocity, density and viscosity and the acoustical parameters calculated from the measured data.

Table 1: The ultrasonic velocity $(\mathrm{U})$, density $(\rho)$ and viscosity $(\eta)$ at various concentrations of 2- (p-methoxy phenyl sulphonyl)-4-methyl sydnone in Ethanol at $303.15 \mathrm{~K}$.

\begin{tabular}{|c|c|c|c|}
\hline $\begin{array}{l}\text { Conc. } \\
\text { (M) }\end{array}$ & $\begin{array}{l}\text { Ultrasonic velocty } \\
\text { U } \\
(\mathrm{m} / \mathrm{s})\end{array}$ & $\begin{array}{l}\text { Density } \\
\rho \\
\left(\mathrm{Kg} / \mathrm{m}^{3}\right)\end{array}$ & $\begin{array}{l}\text { Viscosity } \\
\eta \\
\text { (mPa.s) }\end{array}$ \\
\hline 0.01 & 1201.6 & 801.7 & 0.5333 \\
\hline 0.03 & 1230.7 & 801.9 & 0.5507 \\
\hline 0.05 & 1253.3 & 802.7 & 0.5685 \\
\hline 0.07 & 1274.7 & 803.2 & 0.5918 \\
\hline
\end{tabular}

Table 2: Variation of acoustical parameters at various concentrations of 2(p-methoxy phenyl sulphonyl)-4-methyl sydnone in Ethanol at 303.15K.

\begin{tabular}{lllllll}
\hline $\begin{array}{l}\text { Conc. } \\
(\mathrm{M})\end{array}$ & $\begin{array}{l}\mathrm{Z} \\
\left(10^{3} \mathrm{Kg} / \mathrm{m}^{2} \mathrm{~s}\right)\end{array}$ & $\begin{array}{l}\beta\left(10^{-}\right. \\
\left.{ }^{10} \mathrm{~Pa}^{-1}\right)\end{array}$ & $\left.\begin{array}{l}\mathrm{L}_{\mathrm{f}}\left(10^{-}\right. \\
10\end{array}\right)$ & $\mathrm{r}$ & $\mathrm{R}_{\mathrm{A}}$ & $\begin{array}{l}\tau \\
\left(10^{-10}\right. \\
\mathrm{sec})\end{array}$ \\
\hline 0.01 & 963.322 & 8.6391 & 0.616 & 0.4359 & 1.0292 & 6.1429 \\
0.03 & 986.898 & 8.2333 & 0.602 & 0.4083 & 1.0213 & 6.0454 \\
0.05 & 1006.023 & 7.9311 & 0.590 & 0.3864 & 1.0161 & 6.0118 \\
0.07 & 1023.839 & 7.6623 & 0.580 & 0.3653 & 1.0110 & 6.0460 \\
\hline
\end{tabular}

Table 3: Variation of acoustical parameters at various concentrations of 2 (p-methoxy phenyl sulphonyl)-4- methyl sydnone in Ethanol at 303.15K.

\begin{tabular}{llllll}
\hline $\begin{array}{l}\text { Con } \\
\text { c. } \\
(\mathrm{M})\end{array}$ & $\begin{array}{l}\mathrm{R})^{1 / 3} \\
\left(\mathrm{~m}^{3} / \mathrm{mol}\right)(\mathrm{m} / \mathrm{s}\end{array}$ & $\begin{array}{l}\mathrm{W} \\
\left(\mathrm{m}^{3} / \mathrm{mol}\right)\left(\mathrm{N} / \mathrm{m}^{2}\right.\end{array}$ & $\begin{array}{l}\mathrm{b} \\
\left(\mathrm{m}^{3} / \mathrm{mol}\right. \\
\mathrm{e})\end{array}$ & $\begin{array}{l}\mathrm{V}_{\mathrm{f}}\left(10^{-}\right. \\
\mathrm{m}^{3} / \mathrm{mol} \\
\mathrm{e})\end{array}$ & $\begin{array}{l}\pi_{\mathrm{i}} \\
\left(10^{6}\right. \\
\mathrm{Pa})\end{array}$ \\
\hline 0.01 & 0.6337 & 1.18 & 0.0056 & 0.126 & $\begin{array}{l}6.585 \\
7\end{array}$ \\
0.03 & 0.6864 & 1.27 & 0.0064 & 0.139 & $\begin{array}{l}6.079 \\
2\end{array}$ \\
0.05 & 0.7380 & 1.37 & 0.0072 & 0.151 & $\begin{array}{l}5.661 \\
9\end{array}$ \\
0.07 & 0.7901 & 1.46 & 0.0080 & 0.160 & $\begin{array}{l}5.323 \\
6\end{array}$ \\
\hline
\end{tabular}

(1)

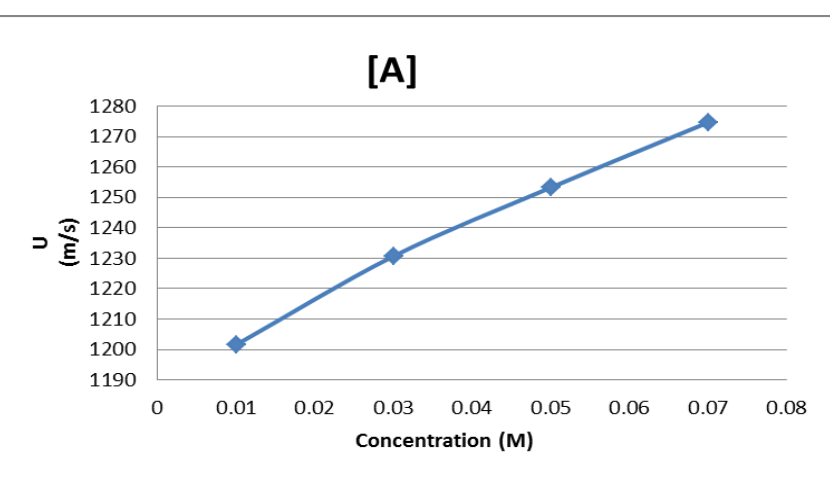

Fig. 1: Variation of Ultrasonic velocity (U) with concentration (M) in Ethanol at $303.15 \mathrm{~K}$

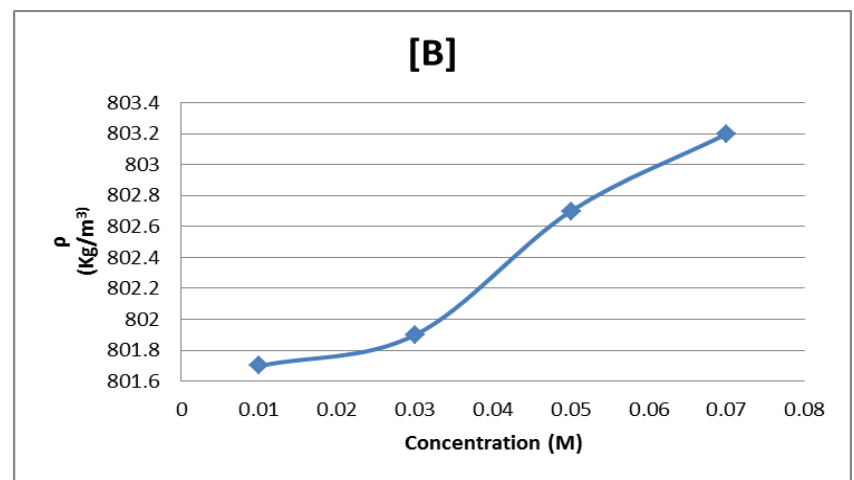

Fig. 2: Variation of Density $(\rho)$ with concentration $(M)$ in Ethanol at $303.15 \mathrm{~K}$. 


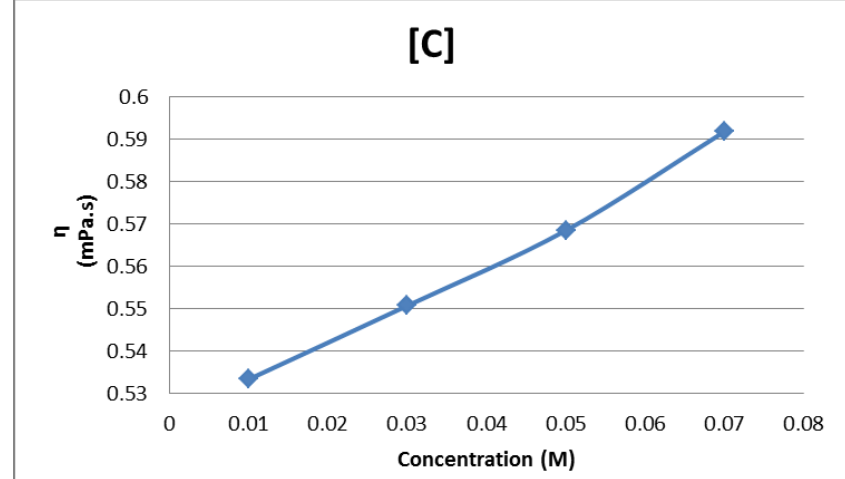

Fig. 3: Variation of Viscosity ( $\eta$ ) with concentration (M) in Ethanol at $303.15 \mathrm{~K}$

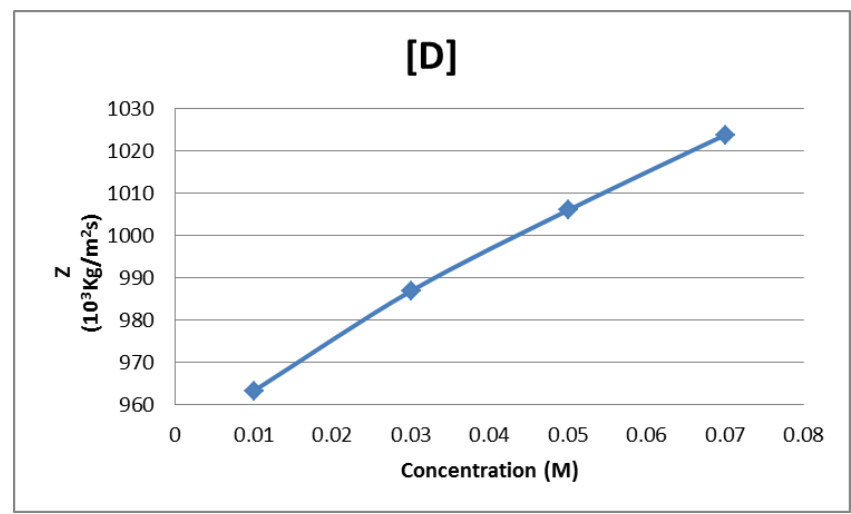

Fig. 4: Variation of Acoustic impedance (Z) with concentration (M) in Ethanol at $303.15 \mathrm{~K}$

\section{[E]}

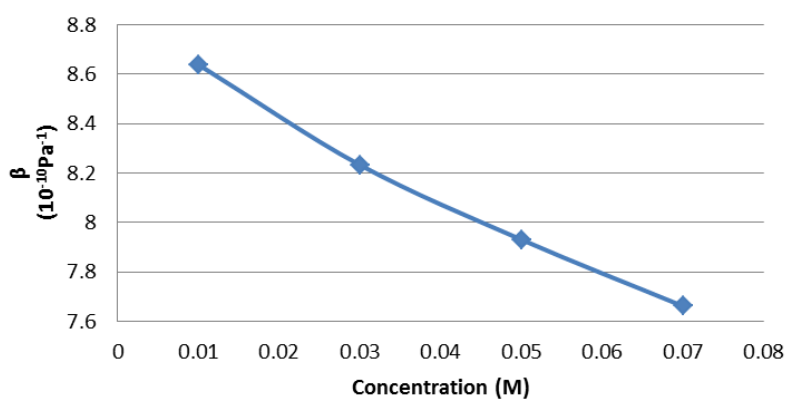

Fig. 5: Variation of Adiabatic Compressibility $(\beta)$ with concentration (M) in Ethanol at $303.15 \mathrm{~K}$.

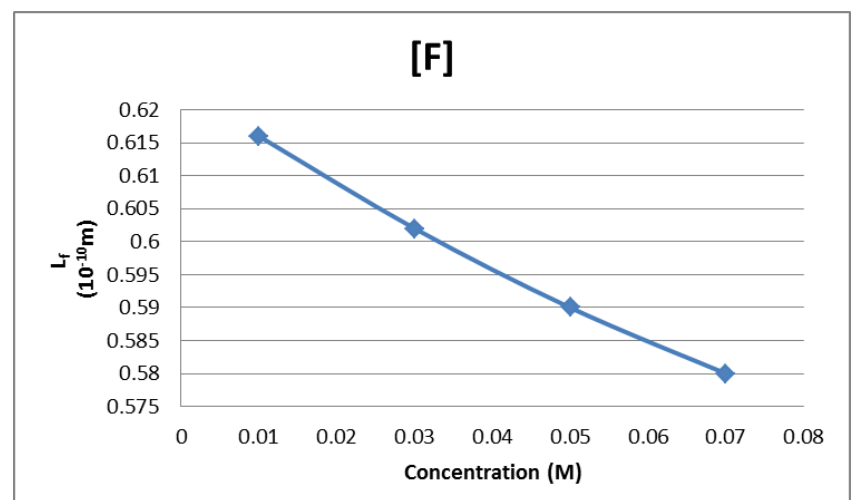

Fig. 6: Variation of Intermolecular free length (Lf) with concentration (M) in Ethanol at $303.15 \mathrm{~K}$.
[G]

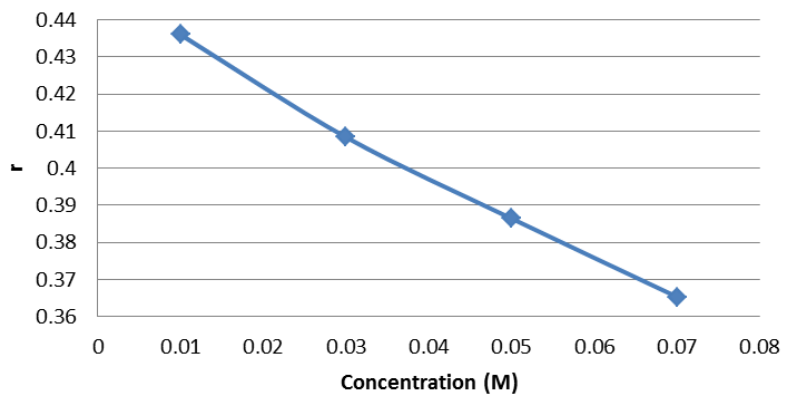

Fig. 7: Variation of Relaxation strength (r) with concentration (M) in Ethanol at $303.15 \mathrm{~K}$.

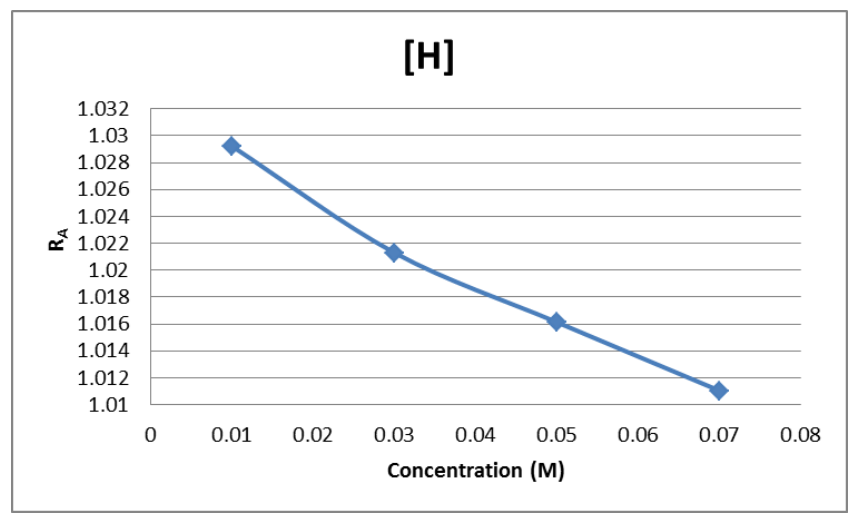

Fig. 8: Variation of Relative association (RA) with concentration (M) in Ethanol at $303.15 \mathrm{~K}$

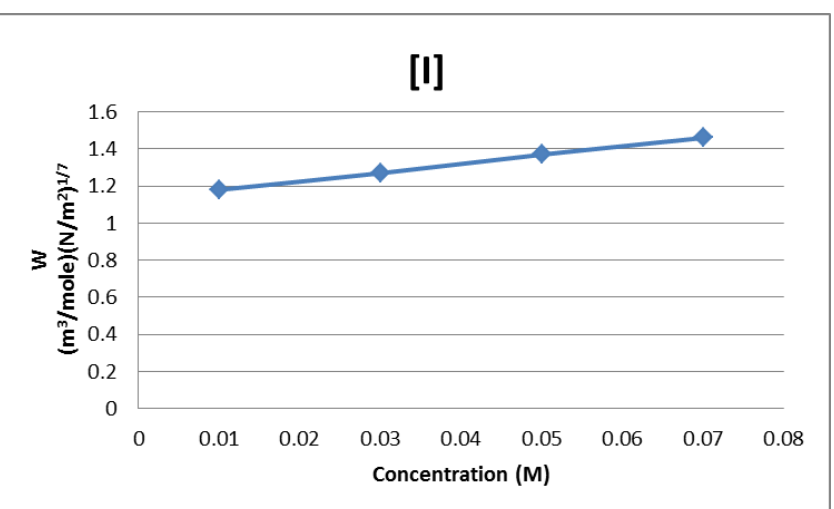

Fig 9: Variation of Molar compressibility (W) with concentration (M) in Ethanol at $303.15 \mathrm{~K}$.

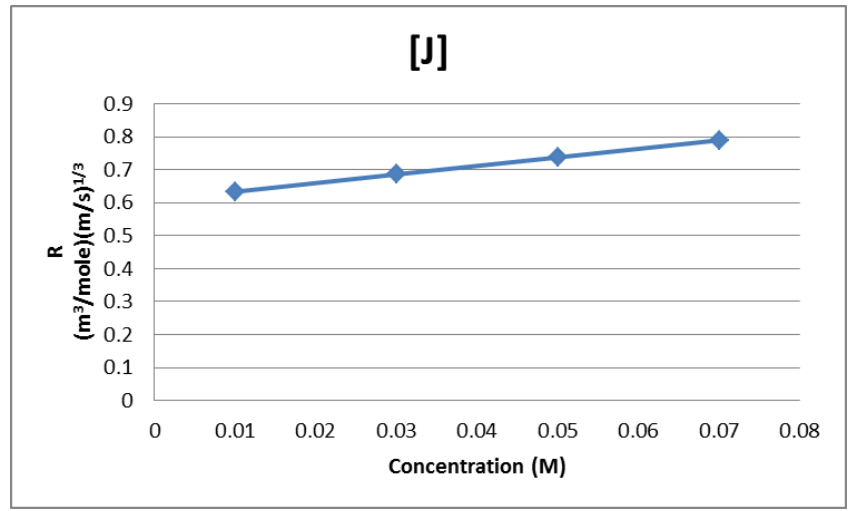

Fig. 10: Variation of Rao's constant (R) with concentration (M) in Ethanol at $303.15 \mathrm{~K}$ 


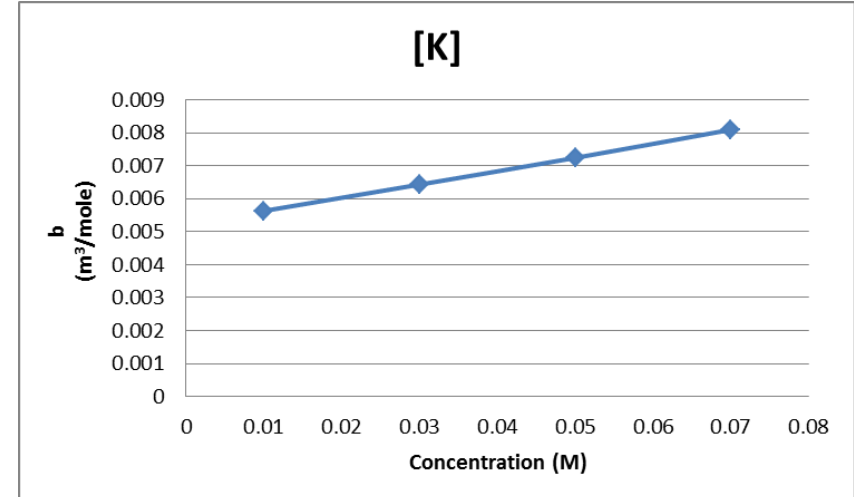

Fig. 11: Variation of Vander Waals constant (b) with concentration (M) in Ethanol at $303.15 \mathrm{~K}$

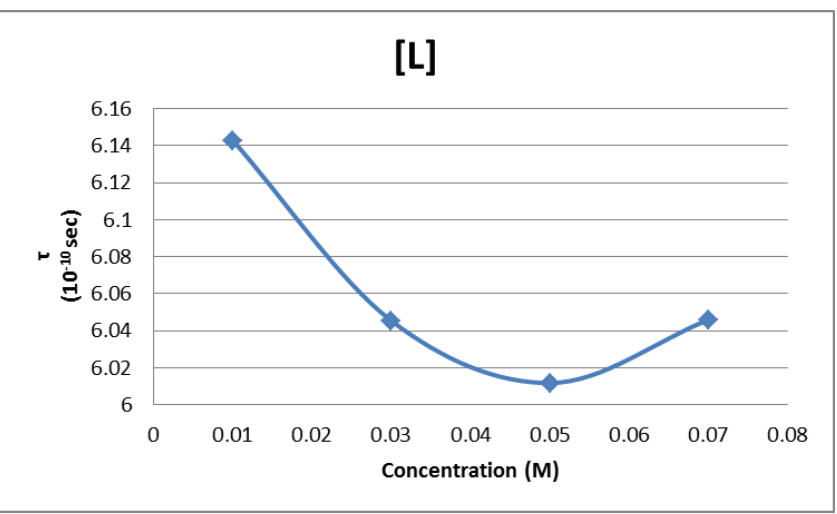

Fig. 12: Variation of Relaxation time $(\tau)$ with concentration $(M)$ in Ethanol at $303.15 \mathrm{~K}$.

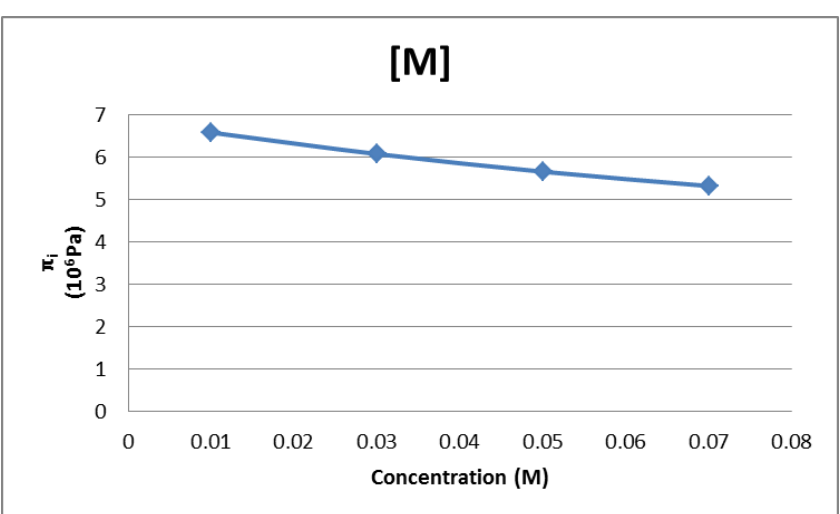

Fig. 13: Variation of Internal Pressure $(\pi \mathrm{i})$ with concentration $(\mathrm{M})$ in Ethanol at $303.15 \mathrm{~K}$.

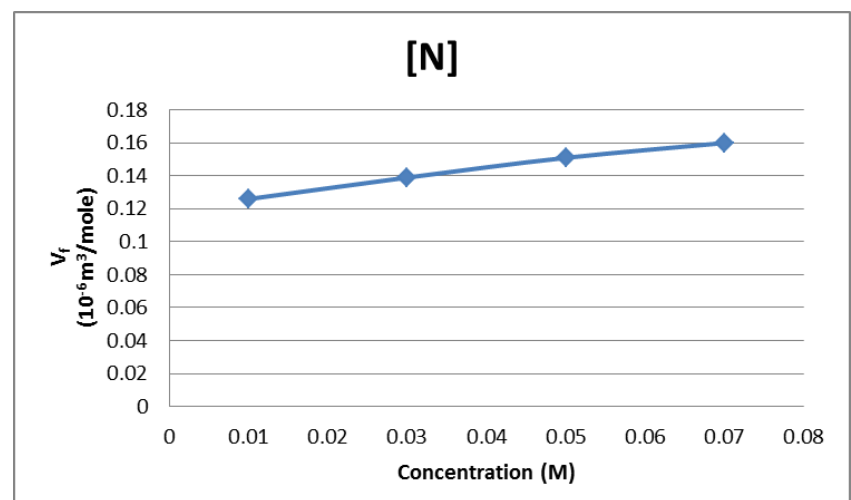

Fig. 14: Variation of Free Volume (Vf) with concentration (M) in Ethano at $303.15 \mathrm{~K}$.

\section{Results and discussion}

Acoustical and thermodynamic properties are of great significance in studying the physico-chemical behaviour and molecular interactions of multi-component liquid mixtures. The molecular interactions existing in the binary liquid mixture are discussed in terms of the acoustical parameters.

Ultrasonic velocity is the speed in which sound propagates in a certain material. It depends on material density and elasticity. Velocity is constant for a given material. Ultrasonic velocity increases with increase in concentration. This increase in ultrasonic velocity suggests powerful solute-solvent interactions. As density increases number of particles in given region is increased, this leads to quick transfer of sound velocity and hence ultrasonic velocity increases with increase in concentration, this result is according to Kharkale ET al24.

Density is an intensive property that increasing the amount of a substance does not increase its density; rather it increases its mass. The density increases with increase in concentration. Increase of concentration results in increase in number of particles in given region which leads to shrinkage in volume of solution and hence density increases with increase in concentration 25 . The viscosity of a solvent or solution is a measure of cohesiveness or rigidity present between either ions (or) ion-solvent (or) solution. The viscosity increases linearly with concentration. This indicates that there exist a strong interaction between solute and solvent.

Adiabatic compressibility is the property of a substance capable of being reduced in volume by application of pressure; quantitatively, the reciprocal of the bulk modulas. The adiabatic compressibility decreases with increase in concentration. Decrease in adiabatic compressibility might be due to aggregation of solvent molecules around solute molecules. This indicates the existence of solutesolvent interactions 26 . The change in adiabatic compressibility in liquid mixtures indicates that there is a definite contraction on mixing and the variation is may be due to complex formation.

Intermolecular Free length denotes the magnitude of either the solute-solute interaction or the solute -solvent interaction or both of the system. Free length decreases with increase in concentration. Decrease in free length is due to compression of liquid, which indicates that the molecules are coming closer to each other; hence the intermolecular cohesion is stronger leading to strong molecular association 27.

According to Eyring and Kincaid28 the regular fall in free length causes a rise in sound velocity in the mixture. This is also in accordance with expected decrease in adiabatic compressibility following an increase in sound velocity. This trend is an indication of clustering together of the molecules into some cage like agglomerates due to associative effect of the polar group predominating over the other type of interactions 29.

Specific acoustic impedance is defined as the impedance offered to the sound wave by the components of the mixture. Acoustic impedance increases with increase in concentration. Increasing trend of acoustic impedance further support the possibility of molecular interaction due to hydrogen bonding between sydnone and alcohol molecule. Specific acoustic impedance is directly proportional to ultrasonic velocity and inversely proportional to adiabatic compressibility and shows similar behaviour to that of ultrasonic velocity and opposite to that of adiabatic compressibility 30 .

Relative association is the measure of extent of association of components in the medium. The Relative association depends on either breaking up of the solvent molecules on addition of solute to it or the salvation of ions that are present. The Relative association decreases with increase in concentration. Decrease in relative association which indicates the breaking up of the solvent molecules on addition of solute 31 .

The Rao's constant and molar compressibility increases with increase in concentration which indicates that the magnitude of interactions is enhanced. This increasing trend of Rao's constant and molar compressibility indicates that availability of more number of components in a given region of space. This leads to tight packing of the medium and thereby increases the interactions 32 . 
Viscous relaxation time is the time taken for the excitation energy to appear as translational energy. The variations in specific relaxation time are mainly due to the change in viscosity of solutions due to both concentration and temperature. The non-linear variation of relaxation time with increase in molar concentration is due to the existence of significant molecular interaction between the solute and solvent molecules.

The term relaxation strength describes several processes by which nuclear magnetization prepared in a non-equilibrium state returns to the equilibrium distribution. The relaxation strength decreases with increase in concentration. The Vander Waals constant increases with increase in concentration.

Cohesion creates a pressure within the liquid of between 103 and 104 atmospheres. This pressure may be regarded as a pressure in the interior of a fluid, resulting from the attractions and repulsions of the molecules and termed as the internal pressure. The internal pressure decreases with increase in concentration, which indicates the decrease in cohesive forces. The reduction in internal pressure may be due to loosening of cohesive forces leading to breaking the structure of the solute. Due to weakening of intermolecular forces of attraction the internal pressure should fall. Decrease in internal pressure indicates that there is a weak interaction between the solute and solvent molecules33.

Free volume is the average volume in which the centre of molecule can move due to repulsion of the surrounding molecules. The free volume increases with increase in concentration. The decrease in molecular association causes an increase in free volume. The increase in free volume may be attributed to lose packing of the molecules inside the shield, which may be brought about by weakening of molecular interactions 34 . Thus free volume is an inverse function of internal pressure. Hence, increase in free volume causes internal pressure to decrease, which indicates the existence of solute-solvent interactions.

Alcohols are strongly self-associated liquids with a three dimensional network of hydrogen bonds 35 and can be associated with any other group having some degree of polar attractions 36 . The associative alcohol molecule act as proton donor enabling hydrogen bonding with sydnone molecule. In the system, studied, the complex formation is likely to occur between $\mathrm{H} \delta+$ of alcohol and $\mathrm{O} \delta$ - of $\mathrm{C}=\mathrm{O}$ group of Sydnone. Hence in the present study there is existence of solute-solvent interactions which are discussed in the above calculated acoustical parameters.

\section{Conclusion}

The Ultrasonic velocity, density, viscosity are measured and related acoustical parameters were calculated from the measured data. The existence of molecular interactions in solute-solvent is favoured in the system, confirmed from the $U, \rho, \eta, \beta, L f, Z \& \pi i$. Hence it is concluded that there exist a molecular interaction between Sydnone and alcohol due to Hydrogen bonding and degree of complexation. Internal pressure gives the idea of the solubility characteristics.

\section{References}

[1] Nikam P S., Jagdale B S., Sawant A B and Mehdi Hasan., Indian J Pure Appl Phys., 2001, 39, 433.

[2] Shipra Baluja and Swati oza., Fluid phase Equilib., 2001, 178, 233

[3] Ali A., Nain A K and Kamil M, Thermochim Acta., 1996, 274, 209.

[4] Manisha Gupta and Shukla J P, Indian J Pure Appl Phys., 1996, 34 772.

[5] Pankaj and Sharma C., Ultrasonics, 1991, 29, 344

[6] Velmurugan S, Nambinarayanan T K., Srinivasa Rao A and Krishnan B., Indian J Phys., 1987, 61B, 105.

[7] Ali A., Abida Hyder S \& Nain A K., Indian J Physics, 2002, 76B (5): 661.

[8] Ali Anwar, Nain A K \& Mohammad Ibrahim, Journal of Pure \& Applied Ultrasonic, 2002, 27: 254

[9] Manohar Murthi N and Nagbhushnam, Indian J Chem., 1984, 23, 510.

[10]Khasare S B., Indian J Pure \& Appl Phys., 1987, 25, 182.
[11]Ali A, Tiwari K, Nain A K and Chakravarthy V, Ind. J. Phy., 2000, 74B (5), 351.

[12]Devadoss.D. D, Thairiyaraja M and Palaniappan L, Ind. J. Phys, 2003, 77B (6), 669-672.

[13]Srinivasulu V \& Naidu P R., J Pure Appl. Ultrasonics, 1995, 17, 14-28.

[14] Kannappan A N \& Rajendran V., Indian J. Pure Appl. Phys., 1991, 29, 465 - 468.

[15]Vibhu I., Misra A., Gupta M., Shukla J D., Ind. J. Phys., 2004, 5, 1147-1155

[16]Pandey J D., Vyas V., Jain G P., Dubey N \& Tripathi., J.Mol.Liq., 1999, 81, 123-133.

[17]Arul G \& Palaniappan L., Indian J Pure Appl. Phys., 2004, 5, 1147 1155 .

[18] Suryanarayana C V., J Acoust Soc Ind., 1983, 13, 11.

[19]Fletcher A., J Phys Chem., 1969, 73, 2217.

[20]Pimental G C and Maclellan A L., The Hydrogen Bond, (W H Freeman and Co, San Fransisco) 1960.

[21] Hobbs M E and Bates W W., J Am chem Soc., 1952, 74, 746.

[22]Hyderkhan V. and Subramanyam S.V., Tras. Parad Soc. (GB) 67, 2282, (1971).

[23] Temperley H.N.V., Rawlinson J.S and Rush brooke G.S., Phys. of simple liquids (John wiley, Newyork), (1968).

[24]Kharkale S S., Wagh M S., Agrawal P S., Paliwal L J., Acoustical studies of Co-polymer of P-Hydroxy Benzoic acid Formaldehyde, Proceedings of Eighteenth National Symposium on Ultrasonics, Vellore 2009; 502-504.

[25] Sunanda et al., International Journal of Pharmacy and Pharmaceutical Sciences, Vol.5, Suppl 1, 76-79.

[26]Patil K.C \& Umare V.D., International Journal of Research in Pure \& Applied Physics, 2012; 2(4): 25-27.

[27]Patil R.A., Dabrase. A., Suryavanshi, B.M., Vidyabharati International Interdisciplinary Research Journal 1(2) 21-26.

[28]Eyring, H. and Kincaid J.F, 1938. Free volumes and free angle ratio of molecules in liquids. J. Chem. Phys., 6:620-629.

[29] Hemalatha, B., 2004. Acoustical behaviour of ternary liquid systems and solids. Ph.D Thesis. Department of Physics, Annamalai University.

[30]Padma S. There., Rasayan J.Chem. Vol. 6/ No.2/111-116/AprilJune/2013.

[31] Shashikant et al., Journal of Chemical and Pharmaceutical Research, 2012, 4(8): 3783-3791.

[32]Nithiyanantham S. and Palaniappan Chem. Sci Trans., 2013, 2(1), 35 40.

[33]Lin W and Tsay S J, J. Phys. Chem, 1990, 74, 1073.

[34] Tadkalkar Alka, Pawar pravina and Bichile G.K, (2011), J.of chem \& phar. Res. 3(3), 165-168.

[35] Rowlison, J.S, (1969). Liquids \& liquid mixtures (2nd Edn) London: Butter worths, 159 (1969).

[36]Ali A, Nain, A.K., and Abida, J. chain. chem. Soc., 51, 477 (2004). 